\title{
Polyethylene three-dimensional nano-networks: How lateral chains affect metamaterial formation
}

\author{
Pedro M. Resende ${ }^{\mathrm{a}}$, Edgar Gutiérrez-Fernández ${ }^{\mathrm{b}}$, Myriam H. Aguirre ${ }^{\mathrm{c}}$, Aurora Nogales ${ }^{\mathrm{b}}$, \\ Marisol Martín-González ${ }^{\text {a,* }}$ \\ ${ }^{a}$ Instituto de Micro y Nanotecnologia, IMN-CNM, CSIC (CEI UAM+CSIC), Isaac Newton, 8, E-28760, Tres Cantos, Madrid, Spain \\ ${ }^{\mathrm{b}}$ Instituto de Estructura de la Materia, IEM-CSIC, C/ Serrano 121, Madrid, 28006, Spain \\ ${ }^{\mathrm{c}}$ Dpto. de Física de la Materia Condensada, Universidad de Zaragoza and Instituto de Nanociencia y Materiales de Aragón (INMA), CSIC-Universidad de Zaragoza, \\ Laboratorio de Microscopía Avanzadas, Edificio I+D - Campus Río Ebro C/ Mariano Esquillor s/n, 50018, Zaragoza, Spain
}

\section{A R T I C L E I N F O}

\section{Keywords:}

Polymer

AAO

Photonic

3D

Nanonetwork

\begin{abstract}
A B S T R A C T
Polymers occupy a central role in current society due to their utility and versatile properties. As part of the class of soft materials, polymers have also been employed in research through nanostructuration and functionalization, looking for the development of new metamaterials with wide applicability in science and society. A particular polymer, Polyethylene, has been widely used both in research and commodities, and new ways to nanostructure and improve its functionality should be considered. Here, we report on the nanostructuration of polyethylene for the fabrication of three-dimensional nanonetworks, by resorting to 3 different variants of polyethylene: low density, high density, and ultra-high molecular weight. Through the melt infiltration of these polymers into well-known three-dimensional interconnected anodic aluminum oxide templates (3D-AAO), a study can be performed to understand the effects of the chain length, structure, and chain-branching of the used polymers on the stability and integrity of the resulting polymeric nanonetworks. This was accomplished by performing infiltrations in the presence of excess bulk material and quenching the infiltration process to access transient infiltration stages. The morphology of these networks was analyzed through SEM and STEM-HAADF to understand the differences arising from polymer structure. The observed results are interpreted through the use of the Lucas-Washburn equation for capillary flow and the determination of the critical contact angle for spontaneous capillary infiltration. The resulting metamaterials also exhibit photonic responses, resulting from the replication of the periodic nature of the employed templates.
\end{abstract}

\section{Introduction}

Polyethylene (PE) represents one of the simplest polymeric materials presently available, with a generic formula $\left(\mathrm{CH}_{2}\right)_{\mathrm{n}}$ [1]. It has been manufactured in small amounts since the 1900s, but the current commercial methods started to be developed around the 1930s. These methods produced low density (LD) PEs, due to the high degree of branching along the main polymer backbone. In later years, other processes were developed for the fabrication of high density (HD) PEs, such as the Philips process. HD PE presents a lower degree of branching, resulting in denser material. The continuous work on the production of PEs allowed for the development of higher molecular weight PEs, of which ultra-high molecular weight (UHMW) PE is an important reference, through the Ziegler-Natta method. These presented long main chains with a high occurrence of chain entanglement, which lowers the polymer density in comparison to HD PEs [1]. PE quickly became an important polymeric material because of its attractive physical and mechanical properties. The regular structure and the absence of strong intermolecular forces that hinder chain mobility, allow the polymer to partially crystallize minimizing its free energy. For this reason, these types of polymers are called semi-crystalline materials. The flexibility of the polymeric backbone and the reduced interactions between different chains also result in lower melting temperatures, making PE an excellent thermoplastic material. In addition to this, the non-polar nature of the polymer chain renders PE resistant to several substances and non-solvents at room temperature. These properties make PE an appealing material in several areas, from industrial applications to commercial products: plastic components and parts; prosthetics and

\footnotetext{
* Corresponding author.

E-mail address: marisol@imm.cnm.csic.es (M. Martín-González).
} 
other health-related applications; packaging of foods, liquids, and hazardous materials; and good thermal and electrical insulation [1,2]. Currently, PE is available presenting different densities and molecular weights, offering different mechanical properties and processability. According to PlasticsEurope, PE-based plastics corresponded to $29.8 \%$ of the total plastic demand in Europe, in 2017, making PE-based polymers one of the most used materials [3]. From the total employed plastic materials, LD, HD, and UHMW PEs are among the most used.

Besides the commercial applications and products, polymers have also drawn special attention in the scientific community as soft materials. These materials exhibit interesting properties at the nanoscale when confinement is of the order of the gyration radius of the polymeric chain $[4,5]$. In the last decadesseveral works have been published for different polymeric materials, studying different phenomena under confined environments such as chain mobility, crystallization, phase transitions, and changes in transport properties [4-14]. PE has also shown interesting properties when confined into long fibers or very small nanowires [15-17]. Other works studied the production of membranes for filtering and separation, mainly membranes for battery technology [18-20]. At the same time, other research groups focused on the use of PE as an enhanced textile material with high breathability and tunable IR transmission [21,22]. And finally, PE has also been reported for the fabrication of composite materials, by a combination of clays, ceramics, or other materials to confer additional properties to the resulting hybrid material [23-25].

The chain structure presented by the polymer also has an impact on its properties. For the particular case of PE, the different grades differ in their degree of branching which has implications on their crystallinity and chain mobility [7,8,26,27]. On the one hand, higher degree of branching results in low polymer crystallinity but also high chain mobility in the melt state, such as in the case of LD PEs. On the other hand, lower degrees of branching confer the polymer high crystallinity but reduced chain mobility, like HD PE's. In addition to chain branching, the molecular weight of the polymer also affects its behavior. UHMW PE presents longer chains when compared with LD and HD PEs, with molecular weights above $10^{6} \mathrm{~g} \mathrm{~mol}^{-1}$ (within the range of 3 to $6 \times 10^{6} \mathrm{~g}$ $\mathrm{mol}^{-1}$ for ultra-high molecular weights). This leads to a higher number of entanglements along the polymer chain hindering chain mobility and increasing the polymer melt viscosity $[2,6,8,28,29]$. Fig. 1 shows a scheme of the polymer chains for LD, HD, and UHMW PE. In bulk, all these materials are semi-crystalline with crystalline lamellae, surrounded by amorphous areas. Depending on the molecular architecture of the PE, the presence of entanglements in the amorphous areas can vary. In this work, we intend to study the behavior of 3 types of PE (LD,
HD, and UHMW) when confined in three-dimensional anodic aluminum oxide (3D-AAO) templates. Previous works have been reported in the literature on the fabrication and use of AAO and 3D-AAO templates for photonics and material nanostructuration [30-35]. In particular, a previous publication from our group has reported the melt infiltration of HD PE in these types of templates, which resulted in 3D PE nanonetworks (NN) with tunable photonic responses [36]. The observed structural coloration in these $\mathrm{NN}$ was associated with the periodic modulation of the refractive index, arising from the anti-replication of the used 3D-AAO templates. The modulation in the refractive index can be interpreted as a result of the layered structure, typically found in distributed Bragg-refractors, that is, the combination of layers with different polymer and air fractions, repeated periodically. The employment of these 3 types of $\mathrm{PE}$ with different densities and chain structure should result in a different organization of the polymer in a finite space as well as changes in the photonic response of the generated NNs. Understanding the behavior of these polymeric NN presents opportunities for their application in areas like separation membranes for batteries, where thinner and porous materials are required for the efficient flow of ions; or sensing applications for fast in situ diagnostics and detection of chemical species $[19,37,38]$.

\section{Experimental Section}

\subsection{Materials}

Aluminum sheets (Al, 99.999\% wt.) were purchased from Advent Research Materials and served as substrates for anodization. HD PE, industrial grade, was purchased from Simona AG, under the commercial name of PE100 natural, with an average sheet thickness of $2 \mathrm{~mm}$; a tensile modulus of $1.1 \mathrm{GPa}$; an average molecular weight, $\mathrm{M}_{\mathrm{w}}$, of $300000 \mathrm{~g} \mathrm{~mol}^{-1}$; and a density of $0.96 \mathrm{~g} \mathrm{~cm}^{-3}$. LD PE and UHMW PE were purchased from Goodfellow, with average sheet thicknesses of 1 and $0.2 \mathrm{~mm}$, a tensile modulus of $0.1-0.3 \mathrm{GPa}$, and $0.2-1.2 \mathrm{GPa}$ and densities of 0.92 and $0.94 \mathrm{~g} \mathrm{~cm}^{-3}$, respectively. UHMW PE had an average $\mathrm{M}_{\mathrm{w}}$ of $9.2 \times 10^{6} \mathrm{~g} \mathrm{~mol}^{-1}$ (determined by the manufacturer, through viscometry). As for LD PE, the manufacturer was unable to provide technical information on the $\mathrm{M}_{\mathrm{w}}$, which we have assumed to be around that of HD PE. All the used polymeric materials have a monomer molecular weight, $\mathrm{M}_{\mathrm{m}}\left[-\mathrm{C}_{2} \mathrm{H}_{4^{-}}\right]$, of $28 \mathrm{~g} \mathrm{~mol}^{-1}$. Acetone and isopropanol were purchased from Carlo Erba Reagents. Ethanol (Absolute) was purchased from PanReac AppliChem. Sulfuric acid $\left(\mathrm{H}_{2} \mathrm{SO}_{4}, 96 \%\right)$ was purchased from J. T. Baker. Perchloric Acid $\left(\mathrm{HClO}_{4}, 70 \%\right)$ was purchased from Alfa Aesar. Copper Chloride $\left(\mathrm{CuCl}_{2}, 97 \%\right)$ was purchased

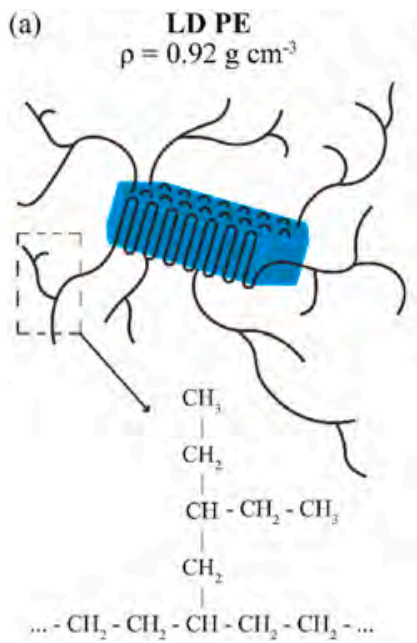

(b)

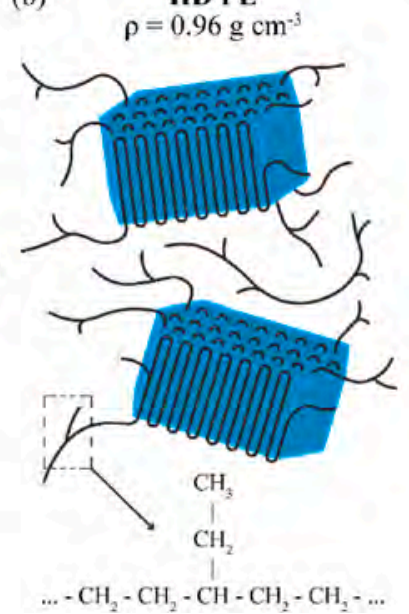

(c)

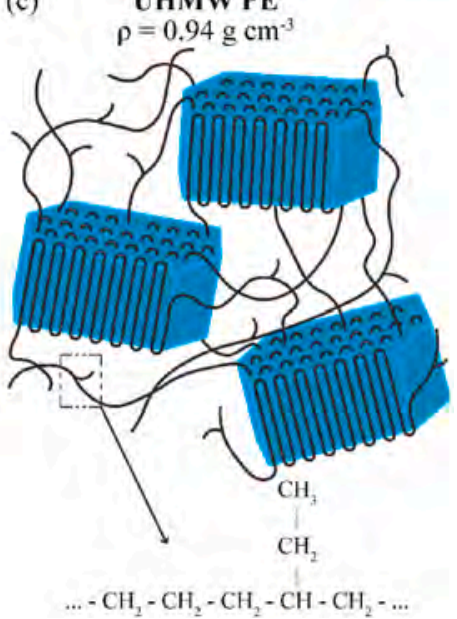

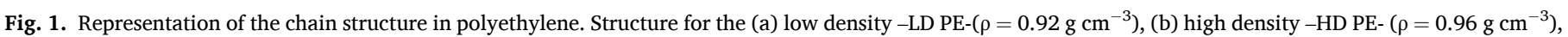
and (c) ultra-high molecular weight -UHMW- $\left(\rho=0.94 \mathrm{~g} \mathrm{~cm}^{-3}\right)$ variants. 
from Aldrich. Orthophosphoric Acid $\left(\mathrm{H}_{3} \mathrm{PO}_{4}, 85 \%\right)$ was purchased from Prolabo.

\subsection{D-AAO templates}

The 3D-AAO templates were produced by the anodic oxidation of $\mathrm{Al}$ substrates, through galvanostatic anodization in a concentrated solution of sulfuric acid, $1.1 \mathrm{M} \mathrm{H}_{2} \mathrm{SO}_{4}, 25 \% \mathrm{v} / \mathrm{v}$ ethanol. This procedure allows for the fabrication of periodically nanostructured templates with tunable photonic responses, by periodically alternating the applied current density. A full description of the anodization and modulation method can be found elsewhere [36]. Before the anodization process, the $\mathrm{Al}$ substrates were cleaned in successive ultrasonic baths of acetone, deionized water, isopropanol, and ethanol, for $7 \mathrm{~min}$ each. After cleaning, the samples were electropolished in a solution of $\mathrm{HClO}_{4}$ and ethanol ( $1: 3 \mathrm{v} / \mathrm{v}, \mathrm{HClO}_{4}$ :Ethanol), by applying a constant potential of 20 $\mathrm{V}$ for $5 \mathrm{~min}$, under vigorous stirring. The substrates were then cleaned with deionized water and dried with an air gun. Each anodization was performed for 3 days in two steps: in the first 1.5 days, the substrates were actively anodized after which they were kept immersed in the electrolyte for the remaining 1.5 days at zero current density. This last step led to the chemical etching of the template due to the acidity of the electrolyte. The gradual etching of these templates throughout the anodization allows the formation of transversal connections that are required for polymer infiltration. This effect has been previously reported for anodization in concentrated solutions [39]. The anodization solution was kept at $-1{ }^{\circ} \mathrm{C}$. The anodized area was limited by the anodization cell, forming a circular area of $c a .6 .327 \mathrm{~cm}^{2}$. After the full anodization process, the samples were removed from the solution and cleaned with deionized water, and dried with an air gun. Before sample use, a surface cleaning method was applied, consisting of the exfoliation of the surface with the aid of a Kapton-tape, effectively removing remains of over-etched aluminum oxide. For this study, 3 template periods were chosen to obtain templates with photonic responses within the red (R), green (G) and blue (B) part of the visible spectrum. Higher template spatial periodicity corresponds to a shift to longer wavelengths (see Supporting Information - SI). Several templates were produced for each color (R, G, and B), with average maxima at 641,553 , and $452 \mathrm{~nm}$ and template out-of-plane periodicities of 267, 220, and $197 \mathrm{~nm}$, respectively. The used method also implies that for longer periods the size of the transversal interconnections is also longer, with values around 97, 68 , and $53 \mathrm{~nm}$ for the R, G, and B samples respectively. An in-plane periodicity can also be defined, which corresponds to the interpore distance, which for this method was around $30 \mathrm{~nm}$. The total thickness of the 3D-AAO templates after preparation and Kapton-tape surface cleaning was around 4-5 $\mu \mathrm{m}$. These values constitute the total template thickness available for polymer infiltration.

\subsection{Polymer infiltration}

For PE infiltration, a melt infiltration method was used. Before infiltration, the samples of LD, HD, and UHMW PE were cleaned in an ultrasonic bath of ethanol for $5 \mathrm{~min}$. The infiltrations were carried out by placing the polymer samples on top of the 3D-AAO templates. The polymer and the templates were kept in a vacuum desiccator chamber with a hot-plate, at low pressure (ca. $100 \mathrm{mBar}$ ) and at a temperature of $170{ }^{\circ} \mathrm{C}$ for $4 \mathrm{~h}$. During infiltration, constant contact pressure was applied between the polymer and the template through the use of a cylindrical metallic weight which flattened the polymer and ensured contact between the template surface and the polymer. The metallic cylinder had an approximate weight of $3.1 \mathrm{~kg}$, applying approximately $33.7 \mathrm{kPa}$ (337 mbar) of pressure, for a total sample diameter of $3.4 \mathrm{~cm}\left(c a .9 .08 \mathrm{~cm}^{2}\right)$. The amount of polymeric material was chosen to ensure that enough material was available to fully infiltrate the templates. Rectangular polymer pieces around $1.5 \times 1.5 \mathrm{~cm}$ and $3 \times 3 \mathrm{~cm}$ were used for HD and LD PE, and UHMW PE respectively, which also took into consideration the thickness of the precursor material. Finally, the samples were removed from the chamber and quenched with a homemade $\mathrm{Al}$ heat dissipater that was kept under $0{ }^{\circ} \mathrm{C}$, effectively stopping the flow of polymer melt. The 3 different template periods were used in the infiltration of each one of the employed polymers, resulting in a total of 9 infiltrated samples. The remaining excess bulk polymer on top of the AAO templates was not removed, serving as support for the generated nanonetworks after template removal.

\subsection{Polyethylene Nanonetworks}

The Polyethylene Nanonetworks were obtained after the infiltration process by removing the remaining $\mathrm{Al}$ and the $\mathrm{AAO}$ template. Firstly, the $\mathrm{Al}$ substrates were chemically removed in an aqueous solution of $\mathrm{CuCl}_{2}$ and $\mathrm{HCl}(10.8 \mathrm{~g}$ and $150 \mathrm{ml}$, respectively, for a total solution volume of $450 \mathrm{ml}$ ). After the complete removal of the remaining $\mathrm{Al}$ the samples were kept in deionized water until the next chemical process. Secondly, the AAO templates were dissolved by selectively etching the samples in an aqueous solution of $\mathrm{H}_{3} \mathrm{PO}_{4}(10 \% \mathrm{wt}$.) at room temperature, for $1 \mathrm{~h}$. Finally, the samples were removed, cleaned with deionized water, and gently dried with an air gun. During drying, strong coloration could be observed, confirming the nanostructuration of the polymer into a 3D nanonetwork. A schematization of this process can be found in the SI, Fig. S1.

\subsection{Free Standing Polyethylene Nanonetworks}

Free Standing (FS) samples of the Polyethylene Nanonetworks were obtained by modifying the previous procedure. After polymer infiltration the excess bulk polymer was manually stripped, exposing the polymer-filled AAO template. The samples were then chemically etched with the same $\mathrm{H}_{3} \mathrm{PO}_{4}(10 \%$ wt.) aqueous solution, at room temperature, for $1 \mathrm{~h}$. The samples were immersed so that the polymer-filled AAO template was facing up. This step was crucial, as the gradual etching of the template releases the generated nanonetworks. The free-standing samples were then removed from the solution and cleaned thoroughly with ethanol. Finally, the samples were left to dry under room temperature conditions. See Fig. S1, in the SI.

\subsection{Characterization techniques}

The different samples were analyzed with SEM, TEM, Spectrophotometry, and Raman spectroscopy. SEM was performed with a FEI Verios 460 HR-SEM. The observations were performed under low current and voltage settings (13 pA and $1.3 \mathrm{kV}$, respectively). Transmission electron microscopy imagining was done in combination with a crioFIB system at liquid nitrogen temperature, to avoid any possible damage from preparation. TEM and STEM-HAADF (High Angular Annular Dark Field HAADF) were performed with a FEI TITAN G2 80-300 kV and a FEI Tecnai F30 $300 \mathrm{kV}$, respectively. CrioFIB was performed with a Cryogenic Dual Beam Nova 200. The FIB system is dedicated to the analysis of soft materials, containing a cryotransfer setup and a cryo-chamber embedded with a sputtering system allowing the analysis of materials at low temperature. Spectrophotometry was performed with a UV-Vis spectrometer from PerkinElmer (Lambda 950 UV-vis). The measured spectra were within the $200-1000 \mathrm{~nm}$ range, after which the maxima of reflection of each sample was extracted. Raman spectroscopy was performed with a Jobin-Yvon LabRam HR using a Nd:YAG laser $(\lambda=532$ $\mathrm{nm})$.

\subsection{X-ray diffraction}

$\mathrm{X}$-ray diffraction was performed to study the crystalline structure and orientation of the PE NN. Measurements were performed with a Philips $\mathrm{X}$ 'Pert PANalytical, using the Bragg-Brentano geometry ( $\mathrm{Cu} \mathrm{K} \alpha$ radiation, $\lambda=0.15418 \mathrm{~nm}$ ) to study the in-plane orientation of the NN. The 


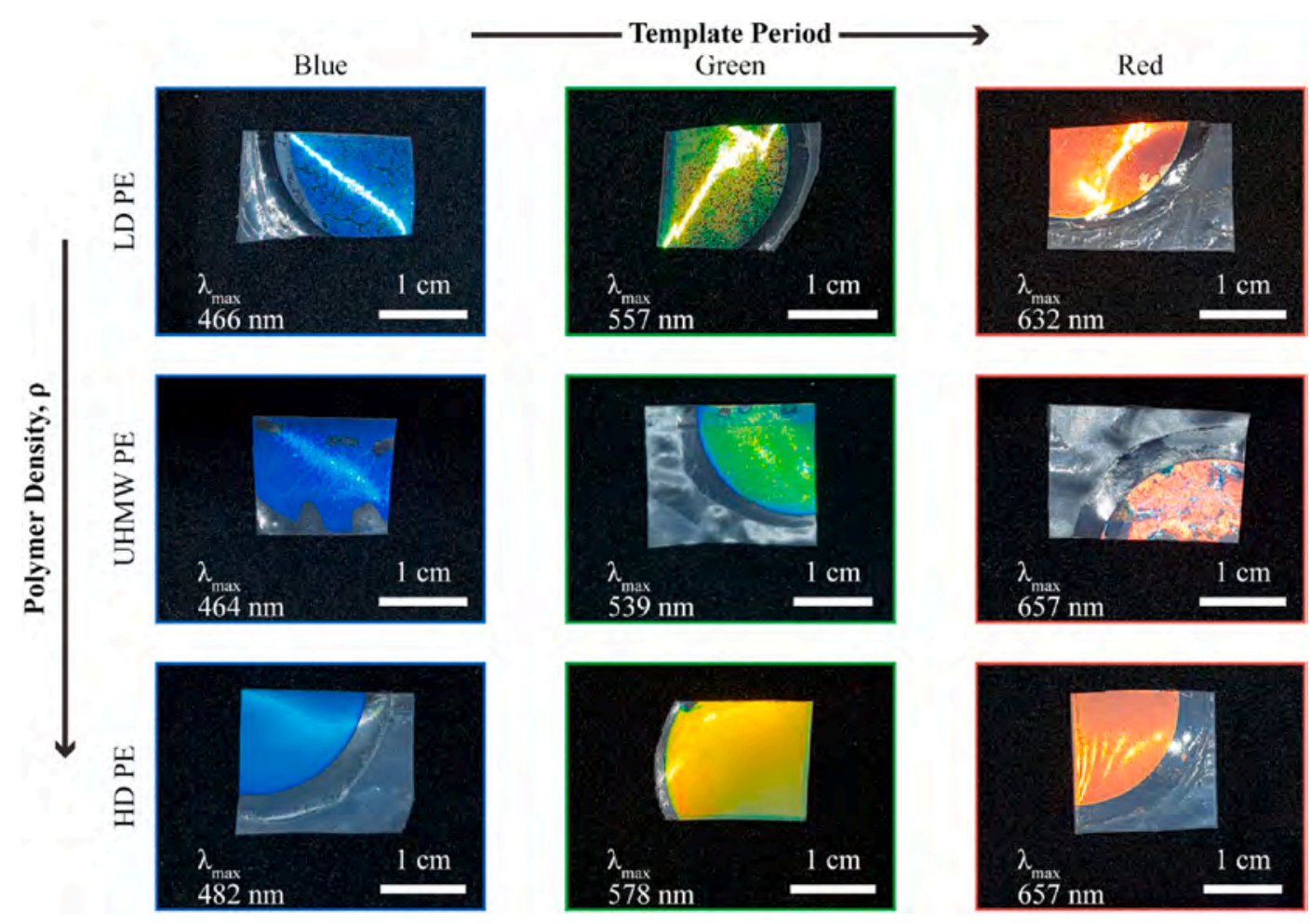

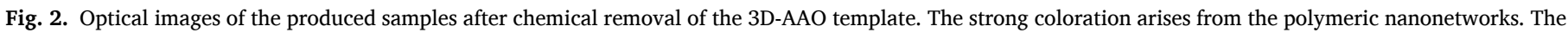
different samples are organized according to the increasing original template period and polymer density.

diffraction vector, $\mathbf{Q}[\mathbf{Q}=(4 \pi / \lambda) \sin (\theta / 2)]$ - where $2 \theta$ is the diffraction angle and $\lambda$ is the wavelength - was oriented normal to the surface of the sample (parallel to the axis of the primary pores or nanorods, hence

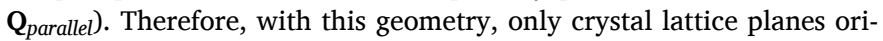
ented parallel to the surface of the template contributed to the diffraction. The out-of-plane measurements were done with Wide Angle X-ray Scattering (WAXS). WAXS was performed at ALBA synchrotron (Cerdanyola del Vallès, Barcelona, Spain) at beamline NCD-SWEET. The Xray beam wavelength was set at $\lambda=0.1 \mathrm{~nm}(\mathrm{E}=12.4 \mathrm{keV})$; the beam size at the sample was $100 \mu \mathrm{m}$ (Horizontal) and $600 \mu \mathrm{m}$ (Vertical). WAXS patterns were collected by a WAXS LX255-HS detector from Rayonix placed at $109.32 \mathrm{~mm}$ from the sample. For this configuration, the scattering vector $\mathbf{Q}$ was nearly parallel to the sample surface, i.e., perpendicular to the long axis of primary pores. In this case, only the crystal lattice planes that are parallel to the nanopore longitudinal axis, contribute to the diffraction pattern.

\section{Results and discussion}

Polymer infiltration was performed with 3 different PE types (LD, HD, and UHMW) that differ in their chain structure and density. Through the use of templates with 3 distinct out-of-place periodicities (having photonic responses in the visible spectrum - R, G, and B), it is possible to study the structural changes of the resulting polymer NNs. The infiltration was performed for the duration of $4 \mathrm{~h}$, after which the samples were immediately quenched, ceasing polymer flow in the template, effectively "freezing" the structures in a transient stage. This allows for a comparison of the obtained structures under the same conditions without reaching a steady-state, which would be represented by a full anti-replication of the template, at an infinite infiltration time. To ensure the outcome was not limited by the amount of material, an excess of the polymer was used in each infiltration. The excess bulk material that remained after infiltration was used as support for the NNs, after the selective removal of the aluminum and the 3D AAO templates. Constant contact pressure was also applied for the duration of the infiltrations to ensure perfect contact between polymer and template (see Experimental Section). The chain structure of the polymer should result in a different organization of the polymer inside these templates, conferring different mechanical behavior and crystallite orientation. The analysis of these samples was divided into major groups: the macroscopic features of the resulting NN, which are also related to their microscopic structure; the influence of polymer structure on the infiltration process; the optical response of the structures and changes in chemical bonds; and finally the crystalline structure of the polymer when molded into the 3D NN.

\subsection{Macroscopic structure and morphology}

After the dissolution of the AAO template, it was possible to observe an immediate change of the photonic response of the samples, displaying strong structural coloration. The samples also presented high flexibility with no damage to the NNs after bending or folding. Fig. 2 shows the different produced samples, originating from the different employed templates, after removal of the 3D-AAO template. Surface morphology was observed to change depending on the polymer type and original template periodicity:

- LD PE presented a non-uniform NN coverage, with visible cracks and peeling of the nanostructured surface film. The surface was seen to improve with increasing template periodicity (from B to R), which can be related to an increase in infiltrated material due to increased thickness of the interconnections in the NN with increasing template period. This results in an overall improvement of NN integrity;

- UHMW PE NNs presented the opposite behavior to that observed for LD PE, in which for the smallest period (B) a uniform macroscopic film was obtained, while for longer template periods $(R)$ the film presented cracks and peeling. This macroscopic observation suggests that, under the same conditions, UHMW PE is unable to fill the template interconnections, resulting in structural damage due to weaker network bonds; 
(a)

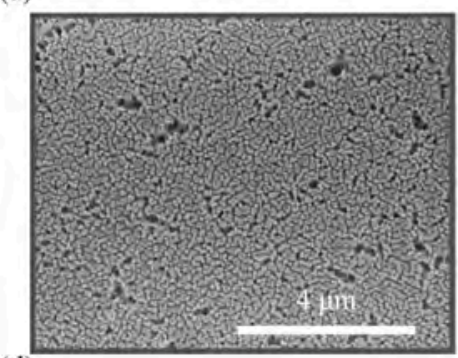

(d)

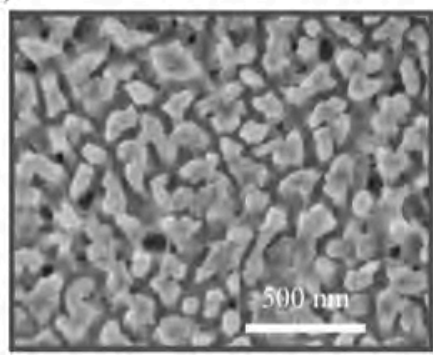

(b)

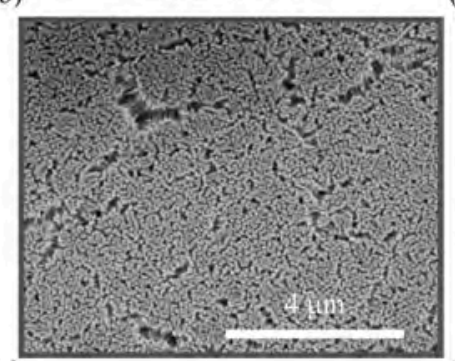

(e)

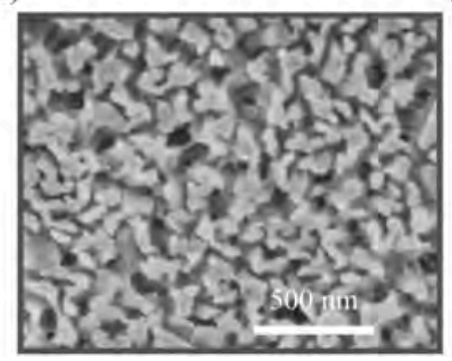

(c)

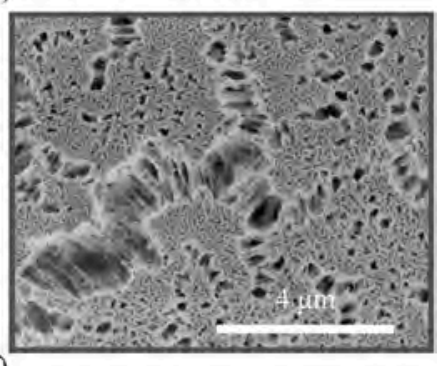

(f)

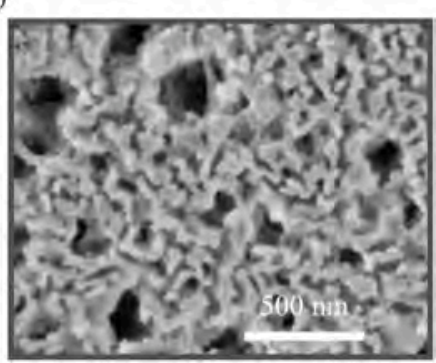

Fig. 3. SEM micrographs of the surface defects for the produced samples after template dissolution. The first row - (a), (b), and (c) - shows images with lower magnification, where the surface crack formation is noticeable. The second row - (d), (e), and (f) - shows topographic details of (a), (b) and (c) respectively, where different interconnected nanowires are visible. The polymers are organized according to increasing polymer density. These networks were obtained from B, R, and $\mathrm{G}$ templates for LD, UHMW, and HD PE respectively. The surface morphology at the microscale was observed to not depend on template periodicity.

- Lastly, the HD PE NNs exhibited the best results, with uniform surfaces and complete coverage of the nanostructured area, with no visible macroscopic damage.

\subsection{Microscopic structure}

The microscopic structure of the samples revealed additional details regarding the organization of the polymer. Fig. 3 shows top-view SEM micrographs of the obtained NNs, for each of the employed polymers. The micrographs in the first row [Fig. 3 (a), (b), and (c)] show examples at lower magnification, where surface defects are visible, such as rifts and openings. The second row [Fig. 3 (d), (e), and (f)] presents details of the micrographs from the first row, showing the interconnected polymeric nanowires. The polymers are presented according to their density (from lower - LD PE, to higher - HD PE). The NNs showed in Fig. 3 were produced from $\mathrm{B}, \mathrm{R}$, and $\mathrm{G}$ templates for the LD, UHMW, and HD PE respectively. The surface morphology at a microscopic level was observed to be the same for the same polymers, independently of the employed template periodicity. An increase in rift/opening formation can be seen with increasing polymer density [Fig. 3 (a), (b), and (c)], also independently of the used template. This behavior is opposite to that observed for the macroscopic structure of the NNs. In this case, the performed observations suggest that, for HD PE, the stress imposed by confinement is locally released through the formation of micro-fractures in the NNs surface. However, for the case of lower density (LD and UHMW), this stress is primarily released at a macroscopic level, with UHMW PE presenting an intermediate behavior between LD and HD PE. From the SEM analysis, it was also seen that the different NNs have similar total thicknesses as the original templates, in this case, around 4-5 $\mu \mathrm{m}$, suggesting the polymer was able to infiltrate until the bottom of the 3D-AAO templates for the used conditions.

These initial observations suggest that the microstructure of the polymeric NNs has a strong dependency on the polymer structure rather than the periodicity of the original templates, and hence the periodicity and thickness of the interconnections in the NNs. In this case, chain mobility should be considered to play an important role in the integrity of the produced NN, mainly on the formation of micro-fractures in HD and UHMW PE. The typical sizes that can be found in this system, for both the nanowires and the different interconnections in the NNs, are in the nanometer scale (average original template pore size of $20 \mathrm{~nm}$ ). This has been previously reported in works dealing with PE infiltration into small diameter AAO templates $[36,40]$. Due to the reduced sizes of these templates, each of the different nanowires present in the PE NNs contains only a small number of polymer chains. This greatly decreases the friction experienced by the different polymer chains, locally enhancing chain mobility. The result is the formation of local cracks and defects due to necking and rupture of different nanowires. From an energetic point of view, the confinement of polymeric chains inside a small space entails an entropic loss due to chain alignment. The removal of the physical constraints (the AAO template) allows the polymeric chains to relax, minimizing the free surface energy. This relaxation is favored by the low inter-chain friction present in the different interconnections as a consequence of the low number of chains.

Despite this, the microstructure of LD PE did not present microscopic fractures or rift-like openings on the surface like in the cases of HD and UHMW PE. The apparent contradiction can be explained by the chain structure that is usually found in low-density polyethylenes. Their low density is attributed to high levels of chain branching, with long sidebranches having sizes of the order of the main chain. The high occurrence of branching in combination with the intricate finite space of the 3D-AAO templates generates LD PE NNs with interlocked polymer chains, with different side-chains moving through different openings inside the template. This additional chain interlocking decreases local mobility and results in local structural integrity (see SI, Fig. S3). The minimization of the free surface energy is still present at a larger scale (macroscopic level) as seen in Fig. 2. For longer template periods (from B to $\mathrm{R}$ ), the reduction of macroscopic fractures and rifts is consistent with an improvement of the structural integrity due to an increase in the amount of polymer present on the interconnections. Nevertheless, the microstructure was observed to be the same independently of the original template period.

To further understand this behavior, cross-sectional images were obtained by cryo-FIB for the 3 types of polymers. In Fig. 4, STEM-HAADF images show 3 different cross-sections for FS nanonetworks produced with the same template period [in this case, the Red (R) templates]. The HAADF is a STEM technique which produces an annular dark-field 
(a)

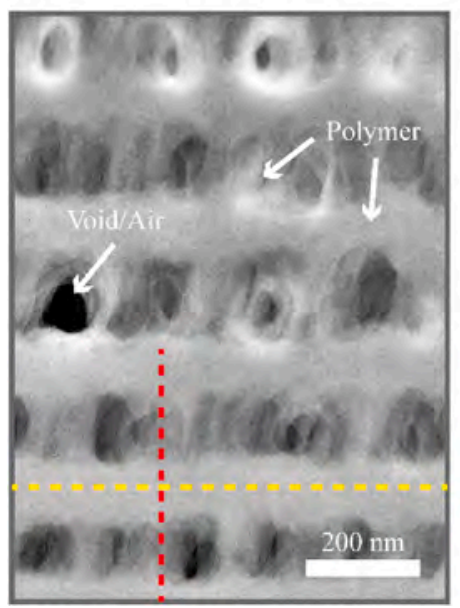

(b)

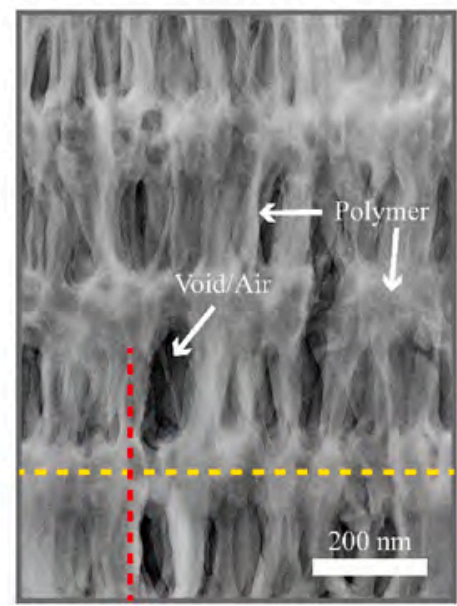

(c)

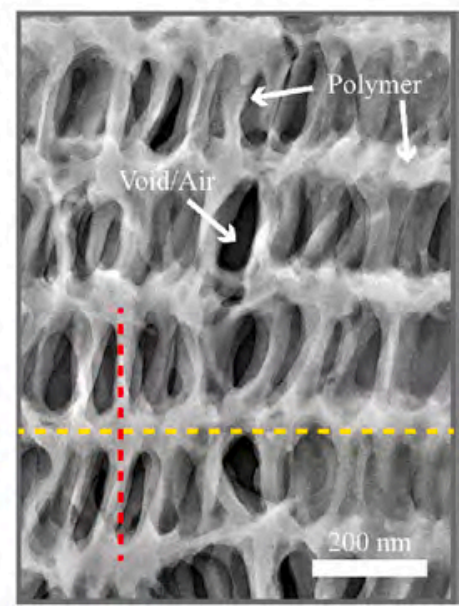

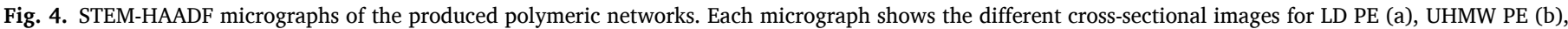

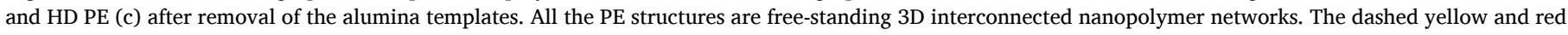

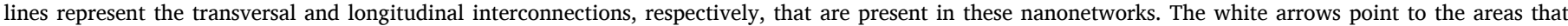

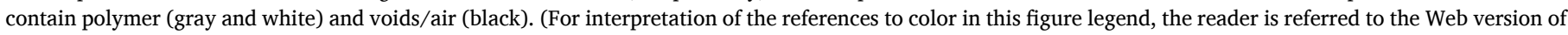
this article.)

image formed by very high angle and incoherently scattered electrons (Rutherford scattered from the nucleus of the atoms). It is highly sensitive to variations in the atomic number in the sample (Z-contrast images) where the intensity is approximately proportional to $\mathrm{Z}^{2}$, the density of material, and the thickness. The contrast micrographs obtained by this method are superior to those obtained from the bright field in conventional TEM, allowing to better resolve the details of the 3D nano-network structure. The 3D nature of the obtained nanonetworks can be readily seen in these micrographs, in which the transversal and longitudinal interconnections are highlighted by the dashed yellow and red lines respectively. For the three cases, it is possible to see morphological changes, particularly regarding the transversal interconnections (dashed yellow lines). For LD PE (a), these connections are visibly thicker and denser than those in UHMW (b) and HD PE (c). From these last two, UHMW PE presents a more porous structure with a fibrous-like morphology. Measuring the average diameter of individual longitudinal segments (dashed red lines) also showed interesting results, with values around 22, 12, and $18 \mathrm{~nm}$ for LD, UHMW, and HD PE respectively. Considering that the average pore size of the employed templates is around $20 \mathrm{~nm}$, the reduced sizes observed for UHMW PE suggest that the infiltration of this variant is less favorable for the same set of conditions, which corroborates the observations of the macroscopic morphology of these NNs. It should also be noted that these results also corroborate the hypothesis that the microstructure is dependent on polymer structure rather than periodicity. At a microscopic level, HD PE presented the most damaged surface, despite having thicker and dense interconnections than UHMW PE. If local intrigrity was solely dependent on nanostructure periodicity, we should have observed UHMW PE to present the most damage and poorer integrity, which was not the case.

\subsection{Influence of polymer structure}

The different results for the 3 different PE densities can also be interpreted through an analysis of the Lucas-Washburn equation for capillary infiltration [41-43]. The equation relates the square of the height of the meniscus, $h$, (in this case of the polymer melt) with time, $t$ :
$h^{2}=\frac{D \gamma_{p} \cos \theta}{4 \eta} t$

In the equation, $D$ is the diameter of the pore, $\gamma_{p}$ is the surface tension of the polymer melt and $\eta$ is the shear viscosity of the melt. In this scenario, where we are using the same template for the three polymers, the shear viscosity represents the most important factor to understand the differences in the melt infiltration. Since $\eta$ takes into consideration the different densities, chain organization and molecular weights. In particular, UHMW PE has a very high molecular weight, $\mathrm{M}_{\mathrm{w}}$, compared to HD and LD PE. Previous studies have shown that $\eta$ is approximately proportional to $\mathrm{M}_{\mathrm{w}}^{3.4}$ for very high molecular weight polymers, while for lower $M_{W}$ values the shear viscosity is seen to have a weaker dependence [44-46]. From this observation, it should be expected that the shear viscosity for UHMW PE, under the same conditions, should be much greater than for HD and LD PE. However, the confinement experienced by the polymer is bound to produce changes in these properties. These changes were observed in previous works on the infiltration of polyethylene oxide (PEO) into AAO templates, where $\eta$ was observed to be approximately proportionate to $\mathrm{M}_{\mathrm{w}}$, instead of the reported $\mathrm{M}_{\mathrm{w}}^{3.4}$ dependence found in bulk materials [47]. This discrepancy between bulk and confined viscosities was addressed by Yao and co-workers through the development of a theoretical model that takes into consideration the existence of an effective viscosity, $\eta_{\text {eff }}$ [47]. Their model was able to explain this discrepancy by demonstrating that for nanopores with a radius much smaller than the polymer gyration radius, $\mathrm{R}_{\mathrm{g}}$, the effective viscosity of the polymer was proportional to $\mathrm{M}_{\mathrm{w}}$.

Even considering a weaker dependence of the viscosity on the molecular weight, the behavior is consistent with the observed results for the infiltration of UHMW PE in the different templates. UHMW PE typically presents molecular weights above $10^{6} \mathrm{~g} \mathrm{~mol}^{-1}$, which are $1-2$ orders of magnitude higher than LD and HD PEs. For the case of the materials used in this work, UHMW PE had a $\mathrm{M}_{\mathrm{W}}$ of $9.2 \times 10^{6} \mathrm{~g} \mathrm{~mol}^{-1}$, which corresponds to approximately a 30 -fold increase from the provided $\mathrm{M}_{\mathrm{W}}$ for HD PE (300000 $\mathrm{g} \mathrm{mol}^{-1}$ ). For longer periods the resulting 3D NNs present several defects at a macroscopic level. For the fabrication method employed for these templates, a longer period is translated into wider transversal interconnections, and thus more available space for polymer infiltration. The increased shear viscosity presented by 
UHMW PE, in combination with the quenching of a transient infiltration stage, prevents the polymer from efficiently filling the available space [see Fig. 4(b)], generating a structure with poor macroscopic structural integrity, despite the observed local structural integrity at the microscale. It has also been observed that branching reduces shear viscosity for low molecular weight polymers $[45,48]$. That is the case of LD PE, which presents significant chain-branching (conferring lower density to the polymer). This behavior is also in agreement with the observed results, as the macroscopic structure is observed to improve for longer template periods (more polymer is infiltrated - see Fig. 4). HD PE exhibited an intermediate behavior as it presents lower molecular weights in comparison to UHMW PE but also lower levels of chain-branching than LD PE (see Fig. 4).

Recently, a work by Ring and colleagues analyzed polymer infiltration by capillary flow with molecular dynamics simulations to understand the effect of confinement on the polymeric chains [42]. In particular, they studied the dependence of the critical contact angle, $\theta_{c}$, for capillary infiltration and showed that $\theta_{c}$ has a weak dependence on chain length, reaching a constant value for large chains. Following the previous results of Yao et al. [47] and a free energy model, an expression for $\theta_{c}$ can be obtained:

$\cos \theta_{c}=\frac{\rho k_{B} T b^{2}}{4 \gamma D}$

In which $\rho$ is the monomer density, $k_{B}$ is the Boltzmann constant, $T$ is the absolute temperature, $b$ is the Kuhn segment length (statistical segment length), $\gamma$ is the surface free energy of the melt and $D$ is the pore diameter. The expression shows a strong dependence of the critical contact angle on the Kuhn segment length (statistical segments) in the polymer chain. The Kuhn segment length can be obtained from the persistence length, $l_{p}$, of the polymer considering the approximation for semiflexible polymer chains through the expression $b \approx 2 l_{p}$. However, the persistence length is a difficult parameter to obtain with only a few experimental and theoretical works reporting some values for different polymers and different chain lengths [49-52]. Nevertheless, qualitative analysis can be performed based on these works and the reported nanostructures to understand polymer behavior. As the infiltrations were all performed under the same conditions, the values for temperature and diameter are constant for the 3 different densities. The surface free energy for PE has been determined both experimentally and through simulations to range between the values of 35.4 and $66.1 \mathrm{~mJ}$ $\mathrm{m}^{-2}$, which are values of the same order of magnitude [53]. For simplicity, it can be assumed that these values have a weak influence on the final value of $\theta_{c}$. From these considerations, we can conclude that the main contributions are from the monomer density and the Kuhn segment length. The monomer density can be estimated from the polymer density and the monomer molecular weight, $\mathrm{M}_{\mathrm{m}}$, through the expression $\rho=\frac{\delta}{M_{m}} N_{A}$, in which $\delta$ is the polymer density and $N_{A}$ is Avogadro's number. The density of the melt is different from the solid density and in this case, the main contribution to the monomer density is the monomer molecular weight. Considering all the used polymers are different variations of Polyethylene, the monomer molecular weight is the same for the 3 cases. The polymer melt density for temperature ranges around the temperature used in this study was observed to be around $0.76 \mathrm{~g} \mathrm{~cm}^{-3}$ for a wide range of molecular weights, both experimentally and in simulation [54]. With the following assumptions the expression for the critic contact angle can be rewritten:

$\cos \theta_{c}=\frac{\delta k_{B} T N_{A}}{4 \gamma D} \cdot \frac{\left(2 l_{p}\right)^{2}}{M_{w}}=\frac{\delta k_{B} T N_{A}}{\gamma D M_{m}} \cdot l_{p}^{2}=C \cdot l_{p}^{2}$

The equation shows that the observed differences should be explained by the persistence length of the polymers. This quantity, in turn, should be influenced by chain length, conformation, and branching. The constant $C$ can be calculated assuming an average melt density of $0.76 \mathrm{~g} \mathrm{~cm}^{-3}$, an absolute temperature of $443 \mathrm{~K}\left(170^{\circ} \mathrm{C}\right)$, an average surface free energy of $51 \mathrm{~mJ} \mathrm{~m}^{-2}$ (considering the reported range of 35.4 and $66.1 \mathrm{~mJ} \mathrm{~m}^{-2}$ ), an average pore diameter of $20 \mathrm{~nm}$ and a monomer molecular weight, $\mathrm{M}_{\mathrm{m}}\left[-\mathrm{C}_{2} \mathrm{H}_{4}-\right]$, of $28 \mathrm{~g} \mathrm{~mol}^{-1}$, obtaining the value of $C$ $=9.8 \times 10^{16} \mathrm{~m}^{-2}$. An estimation of the critic contact angle can be extracted from this expression taking into consideration some published works on the simulation and experimental determination of persistence lengths for PE with different molecular weights and chain branching. Ramachandran et al. [49] reported several values of the persistence length through the use of Small-Angle Neutron Scattering measurements. However, these values are for short branched polyethylene within mid-range molecular weights. These results can still be employed to assess the influence on the critic contact angle. Assuming that $\mathrm{LD}$ and HD PE have a similar molecular weight and differ only on the number of branches per $10^{3}$ carbons, we have chosen the values $\mathrm{M}_{\mathrm{W}}=127 \times 10^{3} \mathrm{~g}$ $\mathrm{mol}^{-1}$ with a polydispersity (PDI) of 5.4 and short-chain branching (SCB) of 3.3 chains per $10^{3}$ carbons for HD PE, and the values $127 \times 10^{3}$ $\mathrm{g} \mathrm{mol}^{-1}, 5.8$ for PDI and SCB of 12.1 chains per $10^{3}$ carbons for LD PE. For these two polymer samples, the persistence length was determined to be 8.2 and $9.0 \AA$ for HD and LD PE respectively. From this data, it is possible to observe that chain branching increases the persistence length of the polymer, as concluded experimentally by Ramachandran et al. [49] Later works on simulation have shown the same tendency, resulting

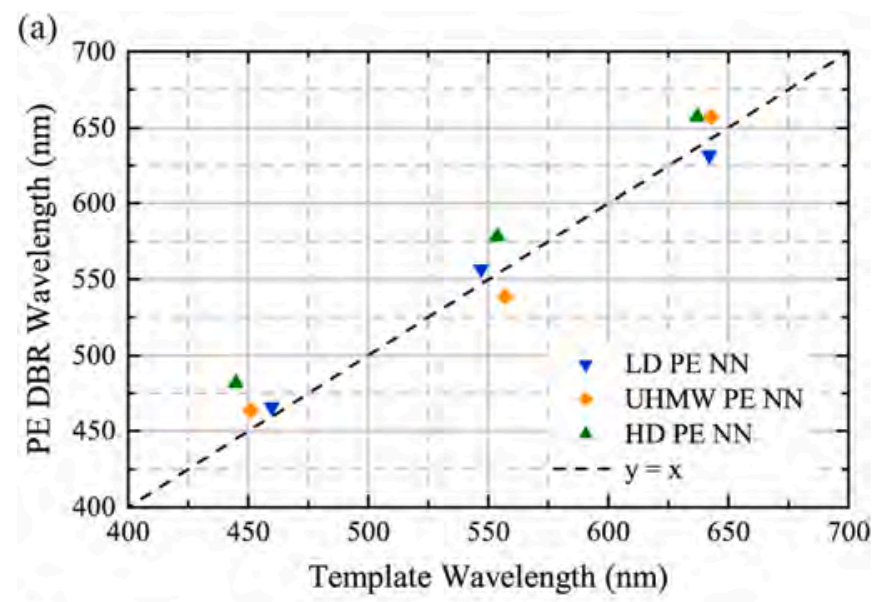

(b)

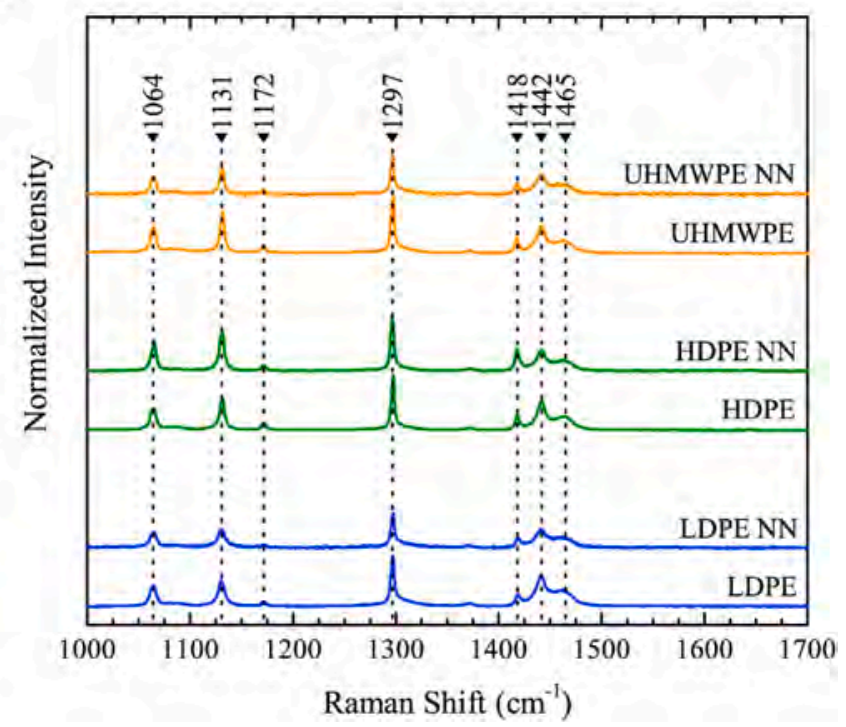

Fig. 5. Spectrophotometry and Raman spectroscopy spectra analysis. (a) Reflection maxima for each of the produced samples versus the employed templates. (b) Raman spectra for the resulting PE NNs and the respective bulk samples. 
in increases in the persistence length with chain-branching [50]. Using these values for the persistence length on the equation above, and solving for $\theta_{c}$, we obtain values of 86.22 and $85.82^{\circ}$ for HD and LD PE respectively. These values are in agreement with the theoretical prediction that longer Kuhn segmental lengths (and so persistence lengths) lead to smaller critical angles, but also in agreement with the experimental results reported here. Under the same infiltration conditions, LD PE presents a smaller contact angle, followed by HD PE and UHMW PE. To the best of our knowledge, there are no available works on the determination of the persistence length for very high molecular weight $\mathrm{PE}$ due to the inherent difficulties of simulating and measuring such long-chain polymeric structures. However, following the results in this work and the calculated approximate values for HD and LD PE we should expect that the persistence length for UHMW PE should be considerably smaller. This trend should be expected considering the relationship between the different physical properties and the polymeric structure of the different polymers. The presence of chain entanglements and the higher crystallinity reported for UHMW PE should be the basis of smaller persistence lengths for long-chained polymers. This is reflected in the experimental data reported here, in which the polymer infiltration and the resulting structures follow the predicted behavior: under the same experimental conditions, LD PE melt infiltration is more favorable, followed by HD PE and lastly UHMW PE that formed a more porous and fibrous nanonetwork. Taking also into consideration the increasing periodicity of the set of used templates, the resulting nanonetworks also follow the same predictions. Long periods mean more available space inside the templates that can be filled with material. As LD PE presents a smaller contact angle and lower viscosity it can fill the templates, improving macroscopic structural integrity with increasing template periodicity (i.e. more infiltrated material that contributes to structural integrity). As for UHMW PE, the viscosity and the expected higher contact angles hinder infiltration, resulting in partial filling of the template, creating a porous $\mathrm{NN}$.

\subsection{Optical characterization}

The photonic response in the visible spectrum was measured to determine the maximum of reflection of each of the produced samples. Fig. 5 (a) shows the reflection maxima of the samples versus the reflection maxima of the employed templates. A guideline is shown for the case where the obtained maximum is equal to that of the template. From the data, it can be seen that the resulting PE NN had only a small shift from the original template maxima. In this case, the absolute average shifts measured for LD, UHMW, and HD PE are of the order of 9, 15 , and $27 \mathrm{~nm}$ respectively. This shift is consistent with the different densities of the employed polymers; in this case, higher densities produce larger wavelength shifts (average densities of 0.92, 0.94, and 0.96 $\mathrm{g} \mathrm{cm}^{-3}$ for LD, UHMW, and HD PE, respectively).

The chemical bonds of the produced PE NN were also studied with Raman spectroscopy and compared with the bulk references. In Fig. 5 (b), a graph is shown with some of the measured spectra for a sample of each type of polymer plus the respective bulk references. From the obtained spectra, no changes were observed between the PE NN and the respective bulk and literature references. [55] No additional peaks were detected, suggesting that the infiltration and subsequent chemical processing of the NN did not introduce any chemical modification to the polymers.

\subsection{X-ray diffraction}

The crystalline structure of the different PE NN was assessed through Wide Angle X-Ray Scattering (WAXS). Considering the size of the polymer macromolecules and the finite space of the template only a few configurations are compatible. To measure the polymer structure without the influence of the template and the bulk polymer, freestanding (FS) samples were specially prepared for these measurements. A detailed description of this process is available in the Experimental Section, and also, in Fig. S2 of the SI. Fig. 6 shows the obtained diffraction patterns for UHMW PE (a) and HD PE (b) NNs and bulk references. The performed WAXS experiments for UHMWPE and HD PE show that the NNs are semicrystalline, and the crystalline phase is similar to that of their corresponding bulk samples. However, they appear to have lower crystallinity, since the ratio between the crystalline reflections and the amorphous halo is smaller in the NNs. Although it was not possible to obtain FS from LD PE, due to the weak structure and integrity of its NNs, WAXS measurements from a powdered sample of LD PE NN were obtained. In these measurements, it was observed that the LD PE NNs are mainly amorphous since its WAXS pattern shows just two halos around the same position as the (1 10$)$ and (2 000$)$ crystallographic planes found in PEs. As mentioned above, the diffraction measurements obtained in the Bragg-Brentano geometry (labeled as XRD in Fig. 6) only the crystallographic planes parallel to the film surface can be detected. This offers some hints about the orientation of the crystals in the pores. As observed, in the XRD measurements, for both samples, the (1 110$)$ and
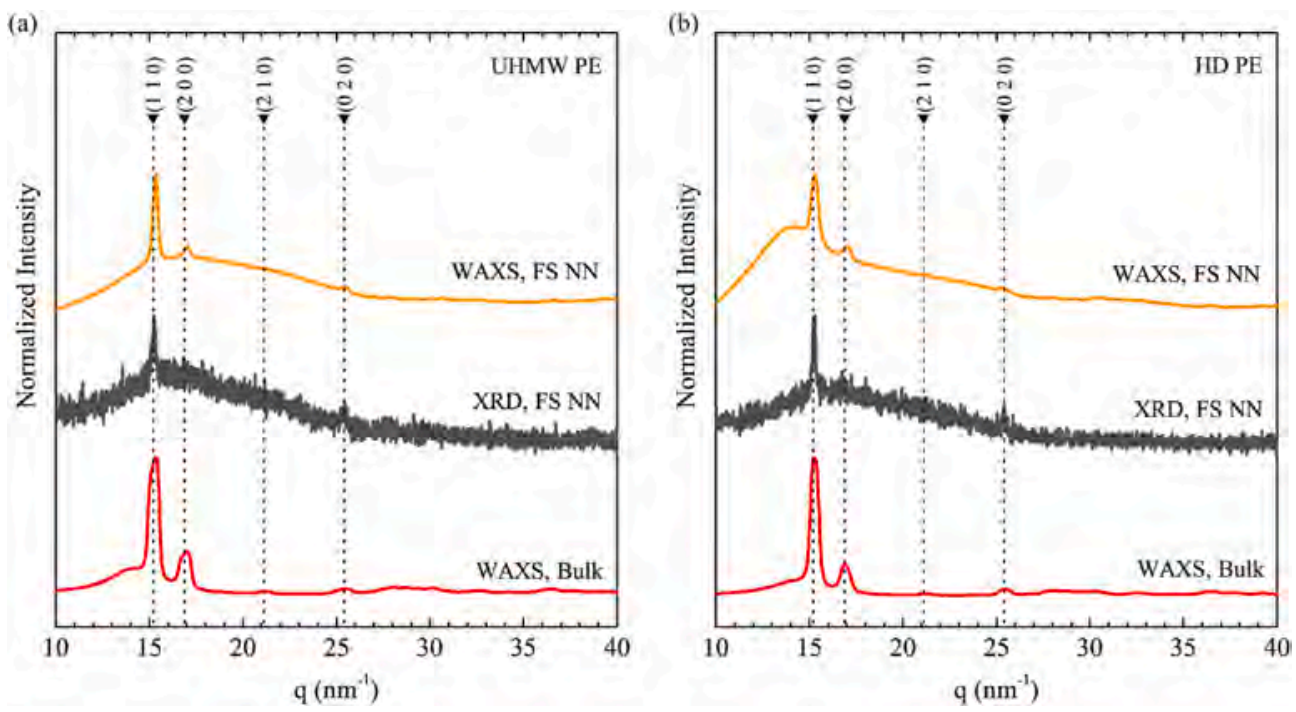

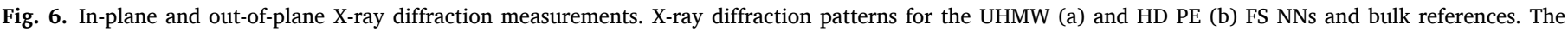
acronym XRD refers to the out-of-plane measurements, while WAXS refers to the in-plane measurements. 
the $\left(\begin{array}{ll}0 & 2\end{array}\right)$ ) peaks are observed, but not the ones corresponding to the (2 $00)$ plane. Although this could be due to the bad signal-to-noise ratio of the measurements, these results could also indicate that melt infiltration produces a kinetic selection of the orientation of the PE crystals along its fastest growth direction (the $b$ direction), similar to what it has been reported for PVDF [56]. Because of that, planes with crystallographic $a$ direction along the primary pore axis are not observed. From the obtained data for UHMW and HD PE, it can be seen that the polymer crystallites are aligned in both directions for both cases, i.e., in and out-of-plane.

\section{Conclusions}

This work reported on the behavior of different types of PE density and structure when confined and nanostructured into a 3D nanonetwork. The infiltration of the polymer with 3D-AAO templates resulted in a 3D structure with strong photonic responses due to the inherent periodicity of the nanostructure, thus, generating structural coloration. This method can be exploited for the fabrication of polymeric Braggrefractor nanonetworks with different mechanical properties, densities, and polymer chain structure. Furthermore, the used methods introduced no detectable chemical modifications or degradation to the polymers. An analysis of the macro and microscopic structure of the generated NNs allowed the understanding of the behavior of the different types of used PE when confined in such complex 3D spaces. The behavior of the NNs is seen to depend not only on the macroscopic properties of the polymer, like the tension modulus but also on the chain organization. On the one hand, due to the lower tension modulus of LD and UHMW PE, the generated NN present macroscopic mechanical failure after template dissolution, unlike HD PE, that presented uniform surfaces with no visible structural damage. On the other hand, the microscopic behavior followed a different trend, showing strong dependence on the chain structure of the polymer. HD and UHMW PE showed microscopic fractures and rifts, which we attributed to a local enhancement of chain mobility due to reduced nanowire size. As for the case of LD PE, the nature of the chain structure conferred additional local structural support, avoiding the formation of local fractures and other defects. The observed behavior of the polymer infiltrations was also discussed in light of the Lucas-Washburn equation for capillary flow. The resulting infiltrations showed a clear dependence on the molecular weight and chain structure of the polymers, with the LD PE samples being able to fully infiltrate the used templates, while the UHMW PE samples (having higher molecular weight) presented loss of structural integrity for longer template periods, due to its higher shear viscosity. Following recent results on the study and simulation of polymer capillary infiltration, an estimation of the critical contact angles, $\theta_{c}$, for spontaneous capillary infiltration was calculated. For the same polymer type (PE), a decrease of the critic contact angle was estimated for highly branched samples (LD PE) in comparison with less branched samples (HD PE). According to this result, it should be expected that very high molecular weight polymers should present shorter persistence lengths when compared with highly branched and lower molecular weight samples as indicated by previous simulation and experimental works. The estimated values for the critical contact angle and the theoretical predictions were in agreement with the observed structures and morphologies. Lastly, X-ray diffraction studies allowed a glimpse into the crystalline structure of the polymers when nanostructured into a $3 \mathrm{D} \mathrm{NN}$, revealing that crystallization took place inside the templates inand out-of-plane with respect to the surface. These results open the possibility for the development of new polymeric materials with direct applications in several areas such as polymer membranes, photonic polymer networks, and flexible polymer sensors as well as guidance on future studies on polymer structure under confinement.

\section{Author contributions}

P.M.R. performed the experiments and sample preparation. P.M.R. also performed optical imaging, SEM, Raman, Spectrophotometry, and X-ray measurements and analysis. E.G.F. and A.N. performed WAXS measurements and analysis. MHA performed the STEM-HAADF experiments and supervised the cryo-FIB lamellae preparation. M.M.G. conceived the idea, supervised the finding of this work, the analysis, and discussed the data. All authors contributed to the final version of the manuscript.

\section{Funding sources}

This work was financially supported by the project MAT2017-86450C4-3-R from MINECO and 2D-MESES from CSIC.

\section{Declaration of competing interest}

The authors declare that they have no known competing financial interests or personal relationships that could have appeared to influence the work reported in this paper.

\section{Acknowledgments}

P.M.R. acknowledges the help of Dr. Eduardo Flores for the X-ray diffraction measurements. The authors acknowledge the service from the MiNa Laboratory at IMN, and funding CM (project SpaceTec, S2013/ ICE2822), MINECO (Project CSIC13-4E-1794), and EU (FEDER, FSE). M. S.M.G wants to acknowledge the projects MAT2017-86450-C4-3-R from MINECO and 2D-MESES from CSIC for financial support. The authors acknowledge the access to the Laboratory of Advanced Microscopy at the University of Zaragoza.

\section{Appendix A. Supplementary data}

Supplementary data to this article can be found online at https://doi. org/10.1016/j.polymer.2020.123145.

\section{References}

[1] S. Ronca, Chapter 10 - polyethylene, in: M. Gilbert (Ed.), Brydson's Plastics Materials (eighth ed.), Butterworth-Heinemann2017, pp. 247-278.

[2] S. Kurtz, Chapter 1-A Primer on Uhmwpe, UHMWPE Biomaterials Handbook, 2009, pp. 1-6.

[3] PlasticsEurope, Plastics - the Facts 2018, PlasticsEurope, 2018. https://www.pl asticseurope.org/en.

[4] D.E. Martínez-Tong, M. Soccio, A. Sanz, C. García, T. A. Fzquerra, A. Nogales, Chain arrangement and glass transition temperature variations in polymer nanoparticles under 3D-confinement, Macromolecules 46 (11) (2013) 4698-4705.

[5] C. Mijangos, R. Hernández, J. Martín, A review on the progress of polymer nanostructures with modulated morphologies and properties, using nanoporous AAO templates, Prog. Polym. Sci. 54-55 (2016) 148-182.

[6] J.F. Vega, S. Rastogi, G.W.M. Peters, H.E.H. Meijer, Rheology and reptation of linear polymers. Ultrahigh molecular weight chain dynamics in the melt, J. Rheol. 48 (3) (2004) 663-678.

[7] Y.F. Yao, S. Rastogi, H.J. Xue, Q. Chen, R. Graf, R. Verhoef, Segmental mobility in the noncrystalline regions of nascent polyethylene synthesized using two different catalytic systems with implications on solid-state deformation, Polymer 54 (1) (2013) 411-422.

[8] S.X. Drakopoulos, G.C. Psarras, G. Forte, I. Martin-Fabiani, S. Ronca, Entanglement dynamics in ultra-high molecular weight polyethylene as revealed by dielectric spectroscopy, Polymer 150 (2018) 35-43.

[9] C. Huang, X. Qian, R. Yang, Thermal conductivity of polymers and polymer nanocomposites, Mater. Sci. Eng. R Rep. 132 (2018) 1-22.

[10] J. Martín, M. Krutyeva, M. Monkenbusch, A. Arbe, J. Allgaier, A. Radulescu, P. Falus, J. Maiz, C. Mijangos, J. Colmenero, D. Richter, Direct observation of confined single chain dynamics by Neutron scattering, Phys. Rev. Lett. 104 (19) (2010) 197801.

[11] B. Sanz, N. Ballard, J.M. Asua, C. Mijangos, Effect of confinement on the synthesis of PMMA in AAO templates and modeling of free radical polymerization, Macromolecules 50 (3) (2017) 811-821.

[12] E. Woo, J. Huh, Y.G. Jeong, K. Shin, From homogeneous to heterogeneous nucleation of chain molecules under nanoscopic cylindrical confinement, Phys. Rev. Lett. 98 (13) (2007) 136103. 
[13] M.M. Rojo, J. Martín, S. Grauby, T. Borca-Tasciuc, S. Dilhaire, M. Martin-Gonzalez, Decrease in thermal conductivity in polymeric P3HT nanowires by size-reduction induced by crystal orientation: new approaches towards thermal transport engineering of organic materials, Nanoscale 6 (14) (2014) 7858-7865.

[14] J. Martín, A. Nogales, M. Martín-González, The smectic-isotropic transition of P3HT determines the formation of nanowires or nanotubes into porous templates, Macromolecules 46 (4) (2013) 1477-1483.

[15] S. Shen, A. Henry, J. Tong, R. Zheng, G. Chen, Polyethylene nanofibres with very high thermal conductivities, Nat. Nanotechnol. 5 (2010) 251.

[16] T. Zhang, T. Luo, Morphology-influenced thermal conductivity of polyethylene single chains and crystalline fibers, J. Appl. Phys. 112 (9) (2012), 094304.

[17] J. Ma, Q. Zhang, A. Mayo, Z. Ni, H. Yi, Y. Chen, R. Mu, L.M. Bellan, D. Li, Therma conductivity of electrospun polyethylene nanofibers, Nanoscale 7 (40) (2015) 16899-16908.

[18] J.-S. Park, S.-J. Gwon, Y.-M. Lim, Y.-C. Nho, Influence of the stretching temperature on an alumina filled microporous high density polyethylene membrane, Mater. Des. 31 (7) (2010) 3215-3219.

[19] T. Knoche, R. Lund, O. Prymak, M. Epple, M. Ulbricht, Effect of annealing temperature on pore formation in preparation of advanced polyethylene battery separator membranes, Materials Today Communications 8 (2016) 23-30.

[20] J. Zuo, S. Bonyadi, T.-S. Chung, Exploring the potential of commercial polyethylene membranes for desalination by membrane distillation, J. Membr. Sci. 497 (2016) 239-247.

[21] P.-C. Hsu, A.Y. Song, P.B. Catrysse, C. Liu, Y. Peng, J. Xie, S. Fan, Y. Cui, Radiative human body cooling by nanoporous polyethylene textile, Science 353 (6303) (2016) 1019.

[22] L. Cai, A.Y. Song, P. Wu, P.-C. Hsu, Y. Peng, J. Chen, C. Liu, P.B. Catrysse, Y. Liu, A. Yang, C. Zhou, C. Zhou, S. Fan, Y. Cui, Warming up human body by nanoporous metallized polyethylene textile, Nat. Commun. 8 (1) (2017) 496.

[23] S. Hotta, D.R. Paul, Nanocomposites formed from linear low density polyethylene and organoclays, Polymer 45 (22) (2004) 7639-7654.

[24] T. McNally, P. Pötschke, P. Halley, M. Murphy, D. Martin, S.E.J. Bell, G.P. Brennan, D. Bein, P. Lemoine, J.P. Quinn, Polyethylene multiwalled carbon nanotube composites, Polymer 46 (19) (2005) 8222-8232.

[25] K.-Q. Yu, J.-T. Yu, J.-G. Dai, Z.-D. Lu, S.P. Shah, Development of ultra-high performance engineered cementitious composites using polyethylene (PE) fibers, Construct. Build. Mater. 158 (2018) 217-227.

[26] L. Wild, T.R. Ryle, D.C. Knobeloch, I.R. Peat, Determination of branching distributions in polyethylene and ethylene copolymers, J. Polym. Sci. Polym. Phys. Ed 20 (3) (1982) 441-455.

[27] W.-S. Tung, R.J. Composto, R.A. Riggleman, K.I. Winey, Local polymer dynamics and diffusion in cylindrical nanoconfinement, Macromolecules 48 (7) (2015) 2324-2332.

[28] Y.-F. Huang, J.-Z. Xu, Z.-C. Zhang, L. Xu, L.-B. Li, J.-F. Li, Z.-M. Li, Melt processing and structural manipulation of highly linear disentangled ultrahigh molecular weight polyethylene, Chem. Eng. J. 315 (2017) 132-141.

[29] W. Li, L. Hui, B. Xue, C. Dong, Y. Chen, L. Hou, B. Jiang, J. Wang, Y. Yang, Facile high-temperature synthesis of weakly entangled polyethylene using a highly activated Ziegler-Natta catalyst, J. Catal. 360 (2018) 145-151.

[30] J. Martín, M. Martín-González, J. Francisco Fernández, O. Caballero-Calero, Ordered three-dimensional interconnected nanoarchitectures in anodic porous alumina, Nat. Commun. 5 (1) (2014) 5130.

[31] P. Resende, R. Sanz, A. Ruiz-de Clavijo, O. Caballero-Calero, M. Martin-Gonzalez Cylindrical three-dimensional porous anodic alumina networks, J Coatings 6 (4) (2016) 59.

[32] A. Ruiz-Clavijo, Y. Tsurimaki, O. Caballero-Calero, G. Ni, G. Chen, S.V. Boriskina, M. Martín-González, Engineering a full gamut of structural colors in all-dielectric mesoporous network metamaterials, ACS Photonics 5 (6) (2018) 2120-2128.

[33] A. Ruiz-Clavijo, O. Caballero-Calero, M. Martín-González, Three-dimensiona Bi2Te3 networks of interconnected nanowires: synthesis and optimization, J. Nanomater. 8 (5) (2018) 345.

[34] T. Kikuchi, O. Nishinaga, S. Natsui, R.O. Suzuki, Fabrication of self-ordered porous alumina via etidronic acid anodizing and structural color generation from submicrometer-scale dimple array, Electrochim. Acta 156 (2015) 235-243.
[35] T. Kikuchi, O. Nishinaga, S. Natsui, R.O. Suzuki, Polymer nanoimprinting using an anodized aluminum mold for structural coloration, Appl. Surf. Sci. 341 (2015) $19-27$.

[36] P.M. Resende, R. Sanz, O. Caballero-Calero, M. Martín-González, Cost-effective, flexible, hydrophobic, and tunable structural color polymeric Bragg reflector metastructures, Advanced Optical Materials 6 (21) (2018) 1800408.

[37] S. Dai, C. Chen, Direct synthesis of functionalized high-molecular-weight polyethylene by copolymerization of ethylene with polar monomers, Angew. Chem. Int. Ed. 55 (42) (2016) 13281-13285.

[38] E.P.A. van Heeswijk, A.J.J. Kragt, N. Grossiord, A.P.H.J. Schenning, Environmentally responsive photonic polymers, Chem. Commun. 55 (20) (2019) $2880-2891$.

[39] Y. Liu, Y. Chang, Z. Ling, X. Hu, Y. Li, Structural coloring of aluminum, Electrochem. Commun. 13 (12) (2011) 1336-1339.

[40] P.M. Resende, M. Martín-González, Sub-10 nm porous alumina templates to produce sub-10 nm nanowires, Microporous Mesoporous Mater. 284 (2019) 198-204.

[41] E.W. Washburn, The dynamics of capillary flow, Phys. Rev. 17 (3) (1921) 273-283.

[42] D.J. Ring, R.A. Riggleman, D. Lee, Critical contact angle to induce capillary rise of polymers in nanopores does not depend on chain length, ACS Macro Lett. 8 (1) (2019) 31-35.

[43] J.L. Hor, H. Wang, Z. Fakhraai, D. Lee, Effect of physical nanoconfinement on the viscosity of unentangled polymers during capillary rise infiltration, Macromolecules 51 (14) (2018) 5069-5078.

[44] T.G. Fox, P.J. Flory, Further studies on the melt viscosity of polyisobutylene, J. Phys. Chem. 55 (2) (1951) 221-234.

[45] W.E. Rochefort, G.G. Smith, H. Rachapudy, V.R. Raju, W.W. Graessley, Properties of amorphous and crystallizable hydrocarbon polymers. II. Rheology of linear and star-branched polybutadiene, J. Polym. Sci. Polym. Phys. Ed 17 (7) (1979) 1197-1210.

[46] D.S. Pearson, G. Ver Strate, E. Von Meerwall, F.C. Schilling, Viscosity and selfdiffusion coefficient of linear polyethylene, Macromolecules 20 (5) (1987) $1133-1141$.

[47] Y. Yao, H.-J. Butt, G. Floudas, J. Zhou, M. Doi, Theory on capillary filling of polymer melts in nanopores, Macromol. Rapid Commun. 39 (14) (2018) 1800087.

[48] R. Ramachandran, G. Beaucage, D.K. Rai, D.J. Lohse, T. Sun, A.H. Tsou, A. Norman, N. Hadjichristidis, Quantification of branching in model three-arm star polyethylene, Macromolecules 45 (2) (2012) 1056-1061.

[49] R. Ramachandran, G. Beaucage, A.S. Kulkarni, D. McFaddin, J. Merrick-Mack, V. Galiatsatos, Persistence length of short-chain branched polyethylene, Macromolecules 41 (24) (2008) 9802-9806.

[50] C. Baig, O. Alexiadis, V.G. Mavrantzas, Advanced Monte Carlo algorithm for the atomistic simulation of short- and long-chain branched polymers: implementation for model H-shaped, A3AA3 multiarm (Pom-Pom), and short-chain branched polyethylene melts, Macromolecules 43 (2) (2010) 986-1002.

[51] H.-P. Hsu, W. Paul, K. Binder, Estimation of persistence lengths of semiflexible polymers: insight from simulations, Polym. Sci. C 55 (1) (2013) 39-59.

[52] I.V. Mikhailov, A.A. Darinskii, E.B. Zhulina, O.V. Borisov, F.A.M. Leermakers, Persistence length of dendronized polymers: the self-consistent field theory, Soft Matter 11 (48) (2015) 9367-9378.

[53] I.-C. Yeh, J.W. Andzelm, Calculations of free energy of surface interactions in crystalline polyethylene, J. Chem. Phys. 149 (1) (2018), 014701.

[54] K.W. Hall, T.W. Sirk, M.L. Klein, W. Shinoda, A coarse-grain model for entangled polyethylene melts and polyethylene crystallization, J. Chem. Phys. 150 (24) (2019) 244901.

[55] H. Sato, M. Shimoyama, T. Kamiya, T. Amari, S. Šašic, T. Ninomiya, H.W. Siesler, Y. Ozaki, Raman spectra of high-density, low-density, and linear low-density polyethylene pellets and prediction of their physical properties by multivariate data analysis, J. Appl. Polym. Sci. 86 (2) (2002) 443-448.

[56] M. Steinhart, P. Göring, H. Dernaika, M. Prabhukaran, U. Gösele, E. Hempel, T. Thurn-Albrecht, Coherent kinetic control over crystal orientation in macroscopic ensembles of polymer nanorods and nanotubes, Phys. Rev. Lett. 97 (2) (2006), 027801. 